\title{
ROTOR SWIVEL MOTOR AS ACTUATOR OF AN INNOVATIVE CONTROL VALVE
}

\author{
M. Sc. Ingo Dietrich*, M. Sc. Lukas Zinßer, M. Sc. Tim Müller, Dr.-Ing. Gerhard Ludwig, \\ Prof. Dr.-Ing. Peter F. Pelz \\ Chair of Fluid Systems, Technische Universität Darmstadt, Otto-Berndt-Straße 2, 64287 Darmstadt \\ * Corresponding author: Tel.: +49 6151 16-27110; E-mail address: ingo.dietrich@fst.tu-darmstadt.de
}

\begin{abstract}
To minimize the complexity of existing process control valves, the authors derived a new control valve design by functional separation and functional integration of functions. One key element of this new valve design is the functional integration of the actuator into the valve's throttle. A hydraulic swivel motor is operated with the process fluid, in this case water. The pressure difference to operate the motor comes from the valve itself. Within this paper we present the control valve design and show in detail how we used the Fail-Early design method to develop the hydraulic swivel motor. The construction is discussed using the experience from manufacturing the prototype and the first experimental results are shown. The experiments yield, that the swivel motor principle is promising. Finally, the next development issues are discussed.
\end{abstract}

Keywords: Swivel Motor, Displacement Actuator, Process Control Valve

\section{INTRODUCTION}

125 different process control valves are listed in the product selector on Samson AG's Homepage [1]. This number is symbolic for the capital goods industry. For customers, a high product individualization with low application complexity (external complexity from product's view) is of steadily increasing, high importance. For manufacturers of fluid components, this results in an enormous number of variants (internal complexity).

The complexity can be attributed to two main characteristics: (i) existing control valves tend to have a dependency between physical and functional structure and (ii) the actuator is sold separately to the valve, requiring knowledge on the customer side to choose the right valveactuator combination.

To reduce the mentioned complexity, we used the methods functional separation and functional integration to derive a new process control valve design with less complexity. A presentation of the new valve design and an evaluation regarding the complexity was published by Dietrich et al. [2]

Of particular interest for the 12th International Fluid Power Conference (IFK) is the functional integration of a hydraulic swivel motor into the valve's throttle. There are two main reasons why the actuator for state-of-the-art globe valves is sold separately from the control valve: (i) the dependency of the needed actuating force on the process pressure and (ii) the available energy source at the valve's installation site, which might be pneumatic or electric. This leads to a complex system from which the customer needs to choose the best fitting product for his requirements.

To overcome the dependency of the needed actuating force (i) on the process pressure we design the seals self-enhancing. To be independent from the available energy source (ii), we use the process fluid itself to operate the swivel motor. Only small pilot valves are necessary to use the existing pressure difference across the valve. Moreover, no high requirements for leakage in the swivel motor are required due to the use of the process fluid.

Within this paper we will give a general presentation of the new valve design, and a more detailed one of the hydraulic swivel motor. During product development we used the FailEarly method to derive a prototype as fast as possible [3]. By testing this prototype, we are able to identify potentials and errors in an early design stage. Thus, closing this paper, we are able to show first experimental data from our prototype 


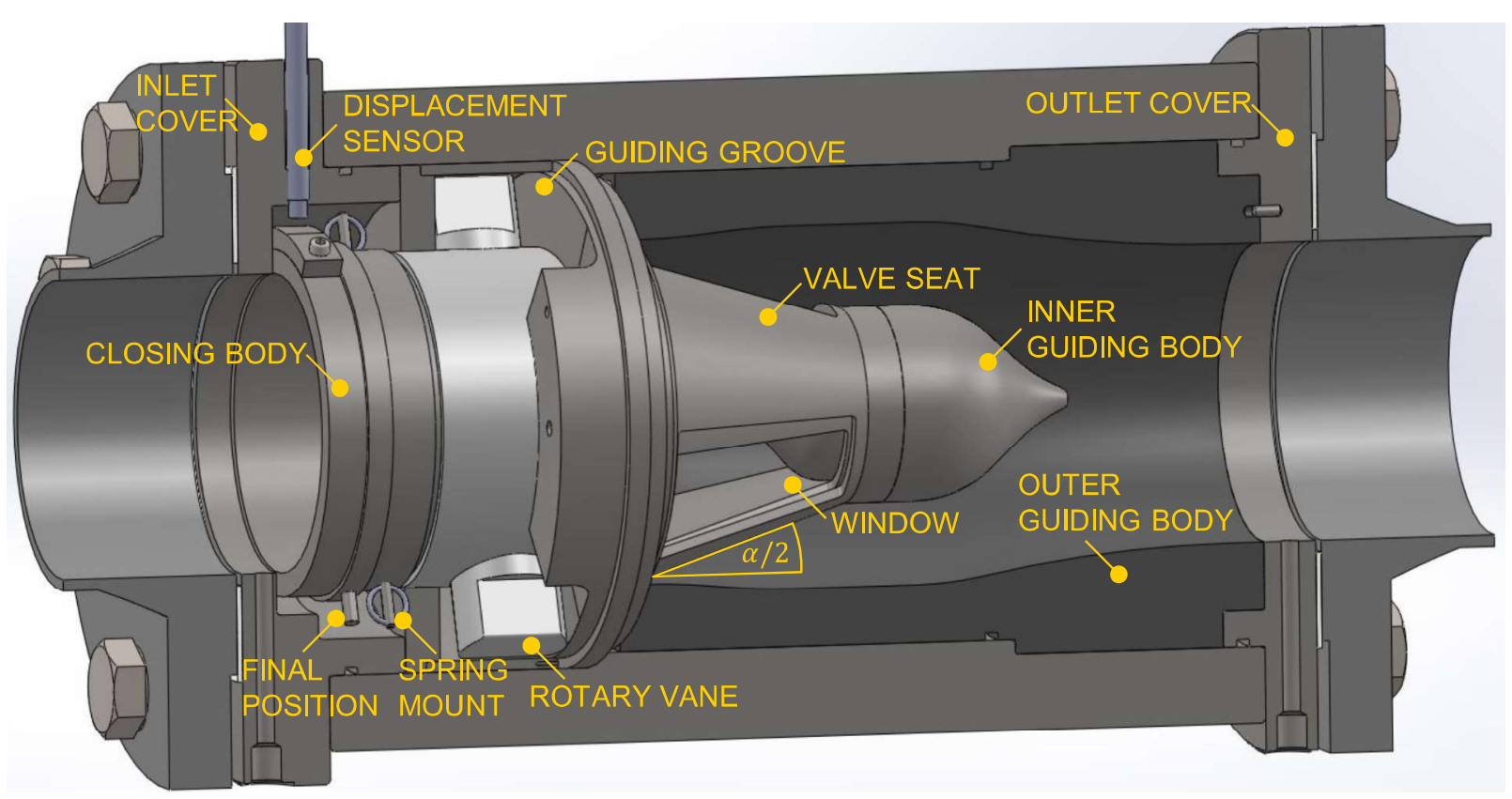

Figure 1: The first prototype of the new process control valve.

and evaluate estimates made during the product development.

\section{NEW VALVE DESIGN}

The first prototype of the developed control valve is shown in Figure 1. It fits the dimensions of a standard DN80 control valve, giving it a length of $310 \mathrm{~mm}$ [4]. An integrated swivel motor is used to change the passed through area of two coneshaped throttle inserts. These throttle inserts are variable, thus allowing different valve characteristics. All components are mounted in a standard housing.

Process fluid enters the valve through a standard flange and gets into the inner movable throttle insert - the closing body. It is located inside of the fixed outer throttle insert - the valve seat. Both, the closing body and the valve seat, contain at least one or more windows, which are passed by the process fluid. The superimposed windows determine the passed through area. This area can be changed by rotation of the closing body. Additionally, the closing body carries out a slight axial movement to achieve an enhanced inner sealing in the valve's closed position.

The cone angle $\alpha$ of both throttle inserts has to be restricted to avoid self-locking while pressing the closing body into the valve seat. Otherwise, a high breakaway force is needed to release the junction between the throttle inserts. Generally, the static friction coefficient $\mu_{0}$, which is crucial for self-locking, has to be determined by doing experiments. Since we decided to exert agile product development, we rely on standard values from literature. To have a conservative estimation we looked for static frictional coefficients in dry environment of the used material combination steel/steel. Found values vary between 0.25 [7] and 0.45 [8], therefore we used $\mu_{0}=0.30$ for our estimation. In case of changing one of the inserts' material to plastic to achieve a better sealing in closed position, the frictional coefficient tends to stay equal or get better. We conclude the cone angle to be

$\frac{\alpha}{2} \geq \arctan \left(\mu_{0}\right)=16.7^{\circ}$.

In order to reduce additional forces on the closing body we choose the cone angle close to the restriction from equation (1) to $\alpha / 2=18^{\circ}$.

The closing body is driven by a swivel motor with rotary vanes in guiding grooves to perform the rotational and axial movement. Process fluid is the operating medium for the rotary vanes. The process fluid is taken from holes in the inlet and outlet cover of the valve. It is guided through a pilot valve and subsequently back into the process control valve. Thus, the differential pressure across the valve is used for the displacement of the rotary vanes and therefore the actuation of itself.

Based on the new valve design we expect lower pressure losses than in conventional globe 
valves across the valve in fully open position because of fewer deflections (see Figure 1). Together with the shape of the windows in the throttle inserts, the inner and especially the outer guiding body have a strong impact on the deflection of the flow. CFD studies have been conducted and found the loss factor of the new throttle valve to be reduced by a factor of approximately seven, in comparison with a globe valve [2]. The pressure losses of the first prototype will be examined at our test rig in Darmstadt in the future.

An inductive displacement sensor is used in combination with an eccentric component to measure the current angle of rotation. The high spatial and temporal resolution of the sensor also allows us to detect vibrations of moving parts. This is a benefit especially for the prototype status. Later on, the expensive inductive displacement sensor could be replaced by a lowcost Hall effect sensor.

The final position for the fully closed and fully open valve is marked by touching dowel pins. Threaded pins in different threaded holes can be used for mounting spring loops of a tension spring. In this way, it is possible to apply a restoring torque on the moving parts in case of blackout.

The first prototype of the throttle valve was designed for operation with water and pressures up to 16 bar.

As already mentioned, a modular design is sought to reduce the internal complexity for the manufacturer as well as the developer and the external complexity for the customer. The modularized structure consists of four different modules: (i) the casing module, (ii) the drive module, (iii) the throttle \& closing module and finally (iv) the guiding module.

\section{HYDRAULIC SWIVEL MOTOR}

The newly developed swivel motor is part of the drive module and can be further divided into the moving rotor and the fixed guiding grooves. It is driven hydraulically by the pilot operated process fluid.

\subsection{Requirements}

Some definite advantages and disadvantages arise, when using process fluid and the differential pressure across the valve to operate the swivel motor. The fact to use process fluid as operating medium for the rotary vanes decreases the necessity for leakage free dynamic sealing as leakage flow does not contaminate the process fluid. On the other hand, the functionality of the swivel motor depends on the available differential pressure. Thus, it is required to achieve low friction in the system to guarantee the possibility to actuate the motor when there is only a small differential pressure available. For high differential pressures, the pilot flow has to be throttled in the pilot valve to reduce the actuating speed.

The rotational and axial movement shall be executed simultaneously and continuous to avoid discontinuities and therefore increase robustness of the drive module. The result is a screw movement of the rotor along the centerline of the throttle valve, which has to be mapped by the guiding grooves.

The requirements in terms of dynamics are relatively small, considering minimal actuating times of 18 to $90 \mathrm{~s}$ [5] for a full opening or closing cycle of a conventional globe valve in ordinary operation. Possible actuating times for emergency operation may reach $4 \mathrm{~s}[6]$.

\subsection{Concept}

For the first prototype, a window design of three quadrangular openings in the closing body and the valve seat is chosen. This results in $120^{\circ}$ action area at the circumference for each window. The expansion of one opening must not exceed $60^{\circ}$ to secure a complete superimposition of the windows in fully open position of the valve and additionally a complete coverage of the windows in fully closed position. Furthermore, the actual expansion of one opening is chosen to be $55^{\circ}$ to establish a $5^{\circ}$-sealing surface for each window in order to enhance the inner sealing in the closed position. The required entire rotational movement is $\gamma=60^{\circ}$ and independent of the sealing surface.

The material combination steel/PTFE is used for every area that is moved and in steady contact to its counterpart to decrease the mentioned friction in the system. The static and kinetic frictional coefficient of this combination are equal, but can vary between $\mu=0.04$ and 0.25 [8]. Due to the equality of those two parameters for this material combination, the stick-slip effect will be prevented. The areas that are moved to 
their counterpart represent the bearing of the closing body and the sealing of the guiding grooves. We omitted separate bearing and sealing, since we want to reduce friction and complexity. In addition, PTFE is traditionally used for both functions.

The movement of the rotor, together with the guiding grooves represent a screw drive, which can be self-locking. The occurrence of selflocking depends on the lead angle $\beta$ of the drive as well as the material of the rotary vanes and the guiding grooves. In this case, self-locking is desirable to prevent the valve from being actuated by the process flow that acts on the pressure effective area of the closing body. The corresponding material combination is steel/PTFE, therefore both, the static and kinetic, frictional coefficient is assumed to be $\mu=0.08$. To achieve self-locking the lead angle has to be

$\beta \leq \arctan (\mu)=4.6^{\circ}$

and is chosen to $\beta=3^{\circ}$.

With the length between the centerline of the rotor and the pressure center of a rotary vane of $l_{\mathrm{rv}}=55 \mathrm{~mm}$ the screw pitch $h$ is calculated to

$h=2 \pi l_{\mathrm{rv}} \tan (\beta)=18.1 \mathrm{~mm}$.

The resulting axial movement $l_{\mathrm{ax}}$ of the rotor during an opening or closing cycle is derived to

$l_{\mathrm{ax}}=\frac{\gamma}{360^{\circ}} h=3 \mathrm{~mm}$.

An estimation for the number of rotary vanes is carried out by calculating the needed drive torque. The performed estimation is uncertain because the friction in the final parts is difficult to predict. According to our development method, the accuracy of the prediction will be determined in the first tests. The most critical case is opening the throttle valve while there is only a small pressure difference $\Delta p_{\mathrm{v}}$ across the valve, because the same pressure difference is used for actuation. The needed torque $T_{\text {stat }}$ for overcoming the static pressure force on the closing body $F_{\text {stat }}$ is calculated and will be compared with the drive torque $T_{\mathrm{d}}$ for a different number of rotary vanes.

The needed torque $T_{\text {stat }}$ depending on the pressure difference $\Delta p_{\mathrm{v}}$

$T_{\text {stat }}\left(\Delta p_{\mathrm{v}}\right)=\frac{F_{\text {stat }}\left(\Delta p_{\mathrm{v}}\right) h}{2 \pi \eta}$ is calculated with the static pressure force on the closing body $F_{\text {stat }}$, the screw pitch $h$ and the efficiency of the screw drive $\eta$. Furthermore $F_{\text {stat }}$ is derived to

$F_{\text {stat }}\left(\Delta p_{\mathrm{v}}\right)=\Delta p_{\mathrm{v}} A_{\text {proj }}$

with the pressure difference $\Delta p_{\mathrm{v}}$ across the valve and the pressure effective area $A_{\text {proj }}$ of the closing body. $A_{\text {proj }}$ corresponds to the area of the nominal diameter minus the three windows of the closing body and a hole for the inner guiding body. The efficiency of the screw drive is calculated to

$$
\eta=\frac{\tan (\beta)}{\tan (\beta+\arctan (\mu))}=39.4 \%[8]
$$

with the lead angle $\beta$ and the frictional coefficient of steel/PTFE. By inserting equation (6) and (7) in equation (5) we receive $T_{\text {stat }}$.

The drive torque $T_{\mathrm{d}}$ is built with the number of rotary vanes $n$, the pressure difference $\Delta p_{\mathrm{v}}$, the pressure effective area of one rotary vane $A_{\mathrm{rv}}$, and the lever between the centerline of the rotor and one rotary vane $l_{\mathrm{rv}}$

$T_{\mathrm{d}}=n \Delta p_{\mathrm{v}} A_{\mathrm{rv}} l_{\mathrm{rv}}$.

The resulting torques are shown in Figure 2. It is visible that for one as well as two rotary vanes the drive torque is always higher than the torque needed to overcome the static pressure difference. Since partially constant friction in the

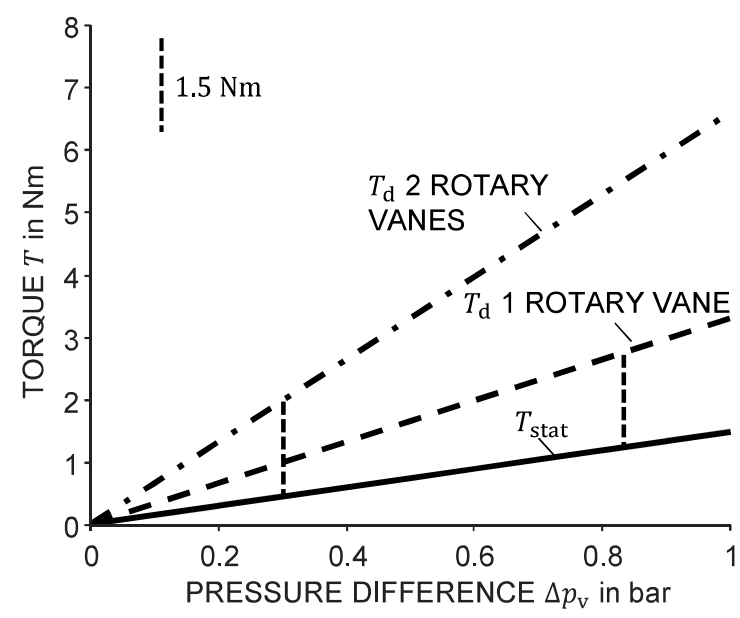

Figure 2: Drive Torque $T_{\mathrm{d}}$ and torque for overcoming the static pressure difference on the closing body $T_{\text {stat }}$ in dependence of $\Delta p_{\mathrm{v}}$. 
system and pressure losses in the pilot control as well as in the swivel motor are not considered in this estimation, the absolute and not the relative difference is crucial. If constant friction and pressure losses resulting in about $1.5 \mathrm{Nm}$ are assumed (as shown in Figure 2), a certain pressure difference across the valve is needed. For one rotary vane, we need at least 0.3 bar and for two rotary vanes at least 0.82 bar. Because we want to have a robust design, we choose $n=$ 2 rotary vanes for the first prototype to secure the actuation also at low available pressure differences. Moreover, two symmetrical arranged rotary vanes, cancel out the radial forces on the closing body. Another possibility to strengthen the robustness is, for example, a larger pressure effective area of the rotary vanes.

A 4/3-way proportional valve has to be used as pilot valve for operating the throttle valve (see Figure 3). The three pilot valve positions are for opening and closing the throttle valve as well as remaining in position. As already mentioned, the pressure difference $\Delta p_{\mathrm{v}}$ is taken from the inlet and outlet cover of the throttle valve. A calculation of the flow coefficient for the pilot valve $K_{\mathrm{VS}, \mathrm{pv}}$ is carried out to ensure proper operation of the throttle valve. The volume flow going through the pilot valve $Q_{\mathrm{pv}}$ is derived with the number of rotary vanes $n$, the pressure effective area of one rotary vane $A_{\mathrm{rv}}$, the travelled path of the rotary vanes along the circumference $l_{\text {tp }}$ and the target actuating time $t_{\mathrm{a}}$ (emergency operation (ref. sec. 3.1) to

$Q_{\mathrm{pv}}=\frac{n A_{\mathrm{rv}} l_{\mathrm{tp}}}{t_{\mathrm{a}}}=0.06 \frac{\mathrm{m}^{3}}{\mathrm{~h}}$.

If a relatively small pressure loss over the pilot valve of $\Delta p_{\mathrm{pv}}=0.25 \mathrm{bar}$ is aspired, the flow coefficient is calculated to

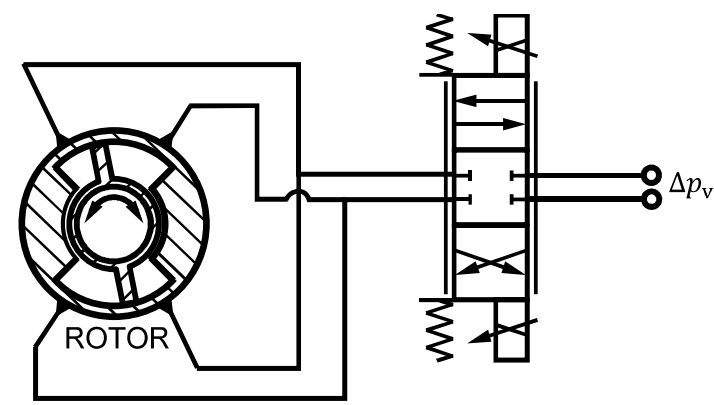

Figure 3: 4/3-way proportional valve as pilot to control the throttle valve.
$K_{\mathrm{VS}, \mathrm{pv}} \geq Q_{\mathrm{pv}} \sqrt{\left(\frac{\Delta p_{1 \mathrm{bar}}}{\Delta p_{\mathrm{pv}}}\right)}=0.12 \frac{\mathrm{m}^{3}}{\mathrm{~h}}$.

The density correction factor in equation (10) is neglected since we are using water as process fluid.

For high available pressures of up to $\Delta p_{\mathrm{v}}=$ 16 bar it has to be granted, that the 4/3-way proportional valve is still in its designated control range, depending on the chosen type of the pilot valve.

\subsection{Design}

Figure 4 shows a halve cut of the guiding groove. It consists of three main parts, the front part, the rear part and the groove base. It is made out of stainless steel and machined by turning and milling. Two pressure line connect to the inlet cover (see Fig. 1), the orientation is ensured by pins. On the right side the valve seat is mounted.

Figure 5 shows the rotor in an exploded view. The two rotary vanes are mounted to the closing body with a pin and a screw. They hold the sliding bushing in its position. The sliding bushing ensures the rotational and axial movement of the closing body. Rotary vane and sliding bushing are made out of PTFE by turning and milling. On the closing body we further mount the excentre, that transforms the angle of

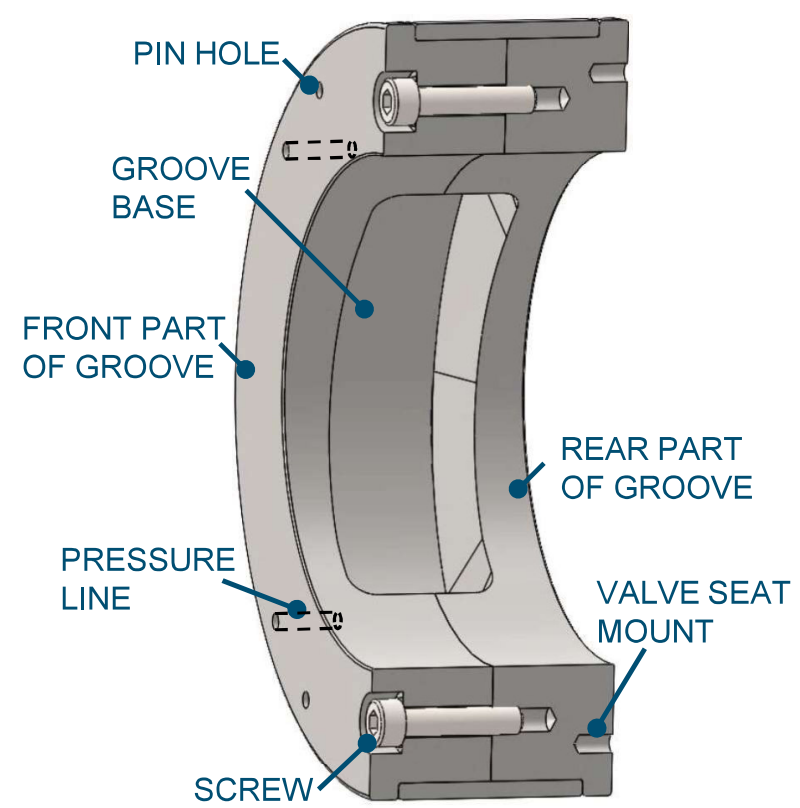

Figure 4: Half cut of the guiding groove. 


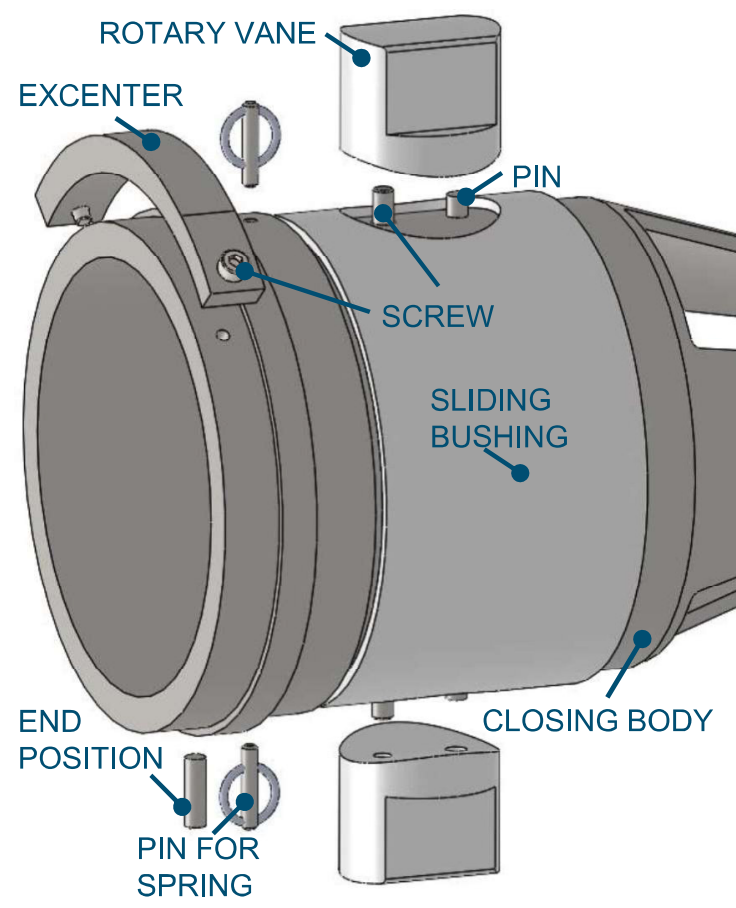

Figure 6: Rotor in exploded view.

rotation into a displacement for our displacement sensor.

Figure 6 shows the working principle of the throttle insert. The rotational and axial movement is visible. In the center position the center line and the edge of the closing body's window are not parallel, due to the necessary $5^{\circ}$ sealing area, that needs to be maintained in the fully closed position. In our design, the rotational angle at which the closing body and the valve seat have solid state contact is determined by the axial manufacturing tolerances. To be able to set it for the first prototype we included a peel plate between the guiding groove and the valve seat. It consists of 10 slices, each being $0.05 \mathrm{~mm}$ thick.

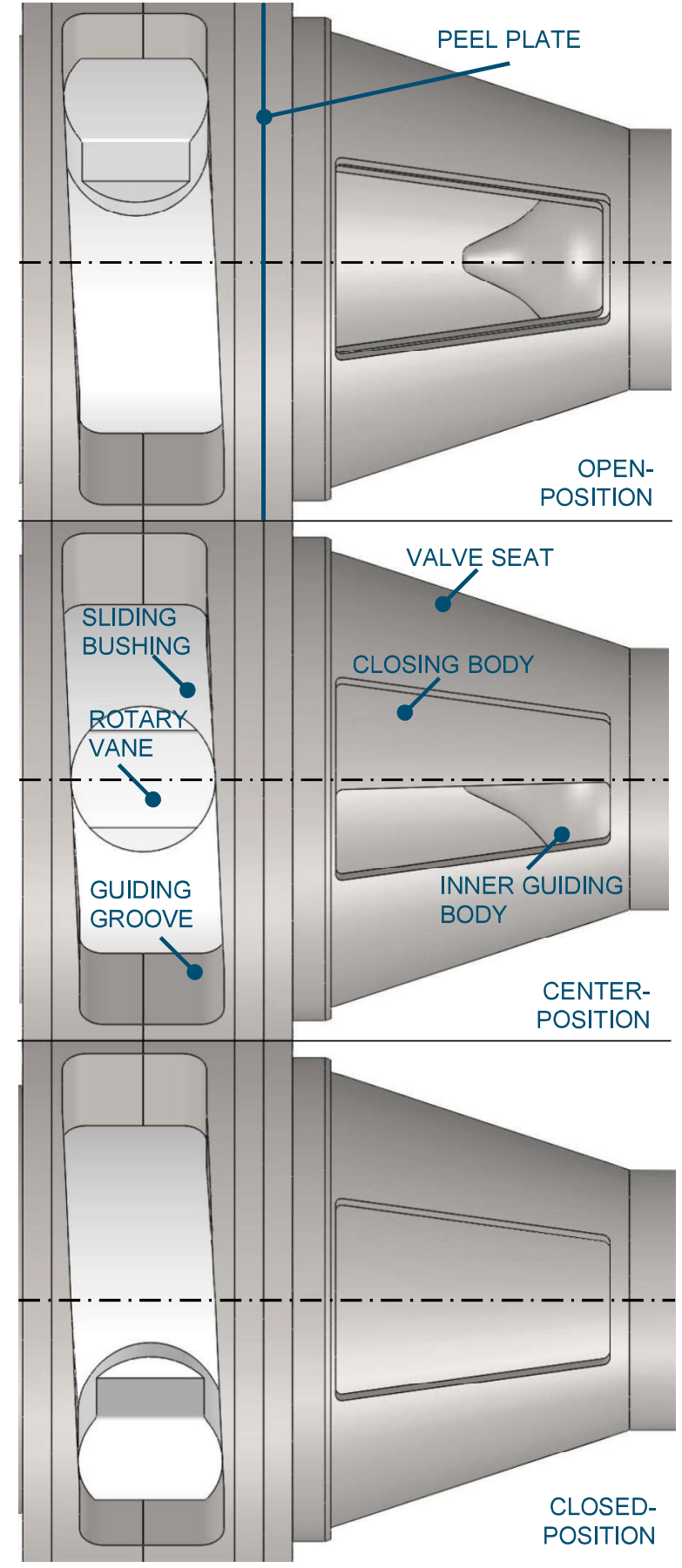

Figure 5: Three positions of the throttle insert.

\section{EVALUATION}

From the manufacturing of the prototype we derived potential improvements for the next development step.

PTFE has a tendency for cold flow/creep which might disqualify it for a long-term operation. Secondly, the sliding bushing is thin walled, with a big diameter. The residual stresses in our PTFE-material made it difficult to stick to 

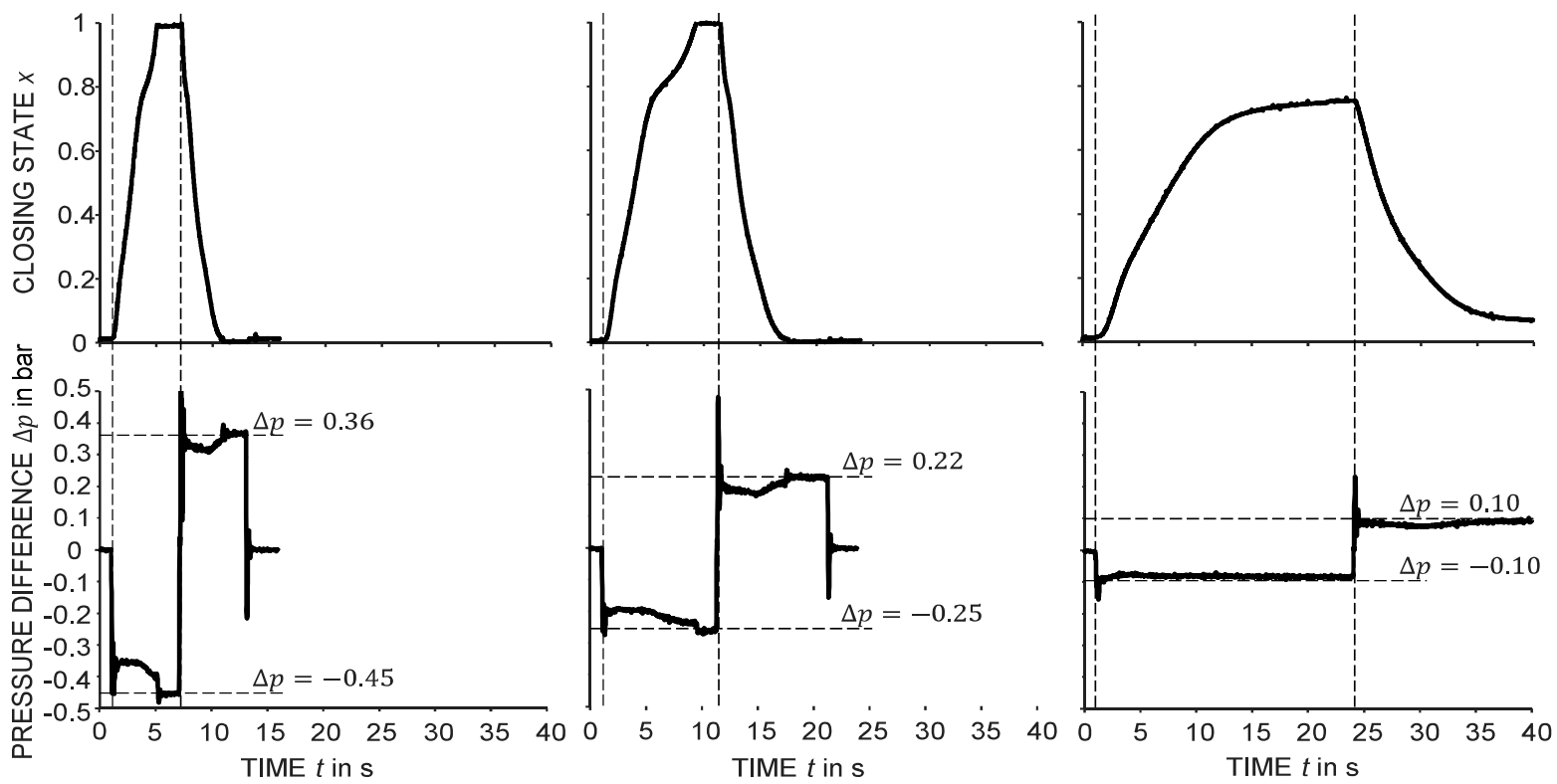

Figure 7: Closing state and pressure difference applied on the swivel motor over time for three different pressure ranges.

the necessary tolerances. Both problems might be tackled by using PTFE with suitable fillers in the next prototype. Finally, the machining of the closing body was challenging, because of the thin walls and the length of the part. Cutting out the windows, alters the tolerances as residual stress deforms the machined parts. A further functional separation of the actuator (consistent of the sliding bushing and the rotary vanes) and the part with the windows might be beneficial.

Another aspect for further improvement is the necessary turndown ratio of the pilot control valve. As it should provide good controllability for high- and low-pressure differences. For our first prototype a suitable 4/3-way proportional valve could not be found. We build the same functionality with one proportional valve and four switching valves.

For the first experimental tests we assembled the prototype as shown in Figure 1. We applied an external, controllable pressure difference $\Delta p$ to our pilot control. To have better insight, we did not mount the process control valve into a piping, but rather placed it in a bucket. This way we could visually inspect the leakage and the movement of the closing body. Proceeding this way, we can ensure, that the kinematic is working, we can evaluate our estimates about the needed torque for actuation and the opening/closing time.

Figure 7 shows the closing state and pressure difference over time for three different pressure ranges. The closing state $x$ equals 0 for the fully open position and 1 for the fully closed position. Since the change of volume for a given change of rotational angle inside the swivel motor is constant and the volume flow rate $Q_{\mathrm{pv}}$ is constant for a constant pressure, one would expect a linear characteristic for the closing state. As Figure 7 shows this is true for the closing state up until $\approx 0.7$, when the slope changes. Since this occurs independent from the pressure difference, we derived that the reason is probably the manufacturing quality of the groove. For the pressure difference $\Delta p=0.1$ bar we can see, that the valve is not able to open and close to the full working range anymore.

Figure 8 shows the time $t$ necessary to close or open the valve for a given pressure difference. The difference for opening and closing most likely comes from our pilot control set up, and different loss coefficients for the piping/hosing for the different states opening and closing. For our design point $\Delta p_{\mathrm{v}}=0.25$ bar we wanted to achieve an operational time of $4 \mathrm{~s}(\mathrm{sec}$. 3.2). In our experiments we were able to open the valve in $5 \mathrm{~s}$ for that pressure difference. Since we neglected any losses in the piping, this is a rather good result.

Figure 9 shows the working range of the swivel motor over the applied pressure difference for an opening of the valve. We can see, that for a pressure difference $\Delta p$ between 0.2 and 0.4 bar a full range operation is possible. For 0.15 bar it 


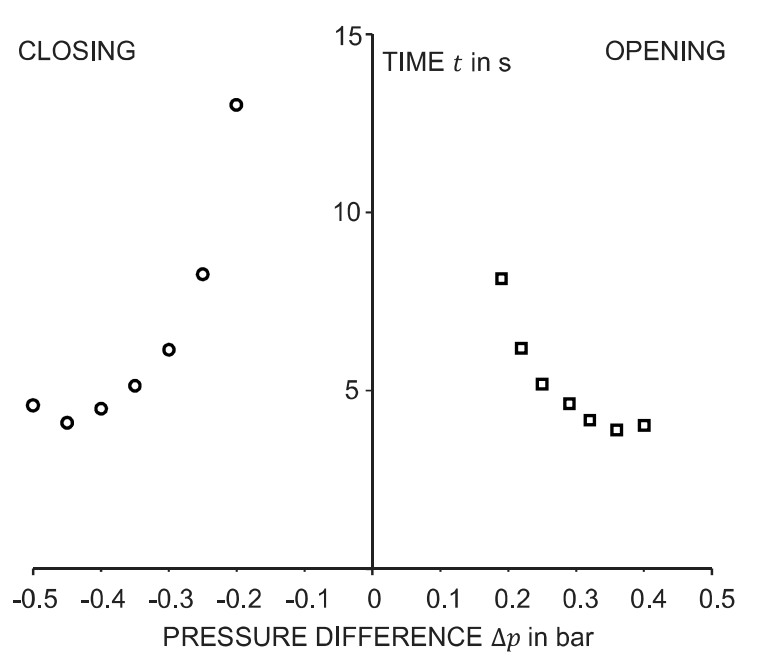

Figure 8: Actuating time of the swivel motor over applied pressure difference.

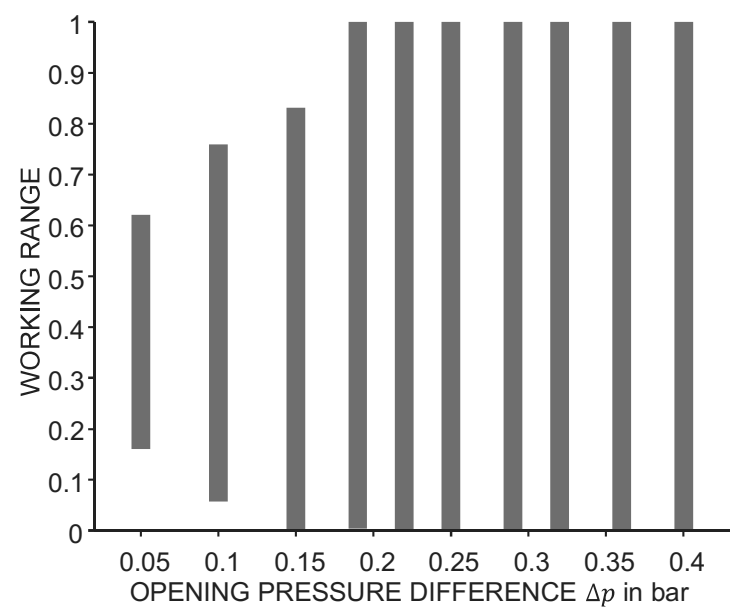

Figure 9: Working range of the swivel motor over applied opening pressure difference.

is not possible to fully close the valve, and below 0.1 bar it is not possible to fully close and open the valve. Using Figure 2, we can derive the friction torque for a given pressure difference $\Delta p$. However, this represents an upper boundary, since there is also a, yet, unknown amount of leakage across the rotary vane driven by this pressure difference. For the minimum working difference $\Delta p=0.2$ bar we read a friction momentum of $1.32 \mathrm{Nm}$.

\section{CONCLUSION AND OUTLOOK}

In this paper we presented a new process control valve design, which we derived through functional separation and functional integration.

Of special interest for the $12^{\text {th }}$ International Fluid Power Conference (IFK) is the functional integration of a hydraulic swivel motor into the valve's throttle. The swivel motor is operated with the process fluid, in this case water, and is made of the material combination PTFE/stainless steel.

We explained our construction in detail and presented the necessary assumptions and calculations during the design phase.

Using the Fail-Early design method, we were able to derive the first prototype within six months from the project's start. First experiments show, that the swivel motor is working almost as designed. We achieve our desired opening/closing time, and the friction within the system is very low. However, we see issues with the manufacturing quality and a different behaviour for opening and closing the valve, which most likely comes from our pilot control set up. With the first prototype we will continue experimental testing. We are especially interested in the valves characteristic and behaviour when it is operating in a flow circuit.

Taking our lessons learned we will improve our design and start building the second prototype. We see three main challenges: i) long term operation of the swivel motor with water or other process fluids in terms of erosion and sedimentation, ii) the necessary turn down ratio of the pilot valve and iii) the long-term behaviour of the PTFE parts.

\section{NOMENCLATURE}

$A_{\text {proj }}$ pressurized projected area of the closing body $A_{\mathrm{rv}} \quad$ pressurized projected area of one rotary vane

$F_{\text {stat }} \quad$ force to overcome static pressure on the closing body

$h \quad$ screw pitch of the drive

$K_{\mathrm{VS}, \mathrm{pv}}$ flow coefficient of the pilot valve

$l_{\mathrm{ax}} \quad$ axial movement of the rotor

$l_{\mathrm{rv}} \quad$ length between the centreline and the rotary vane

$l_{\mathrm{tp}} \quad$ travelled path of the rotary vanes

$T_{\mathrm{d}} \quad$ drive torque of the rotary vanes

$T_{\text {stat }} \quad$ torque to overcome static pressure on the closing body

$t_{\mathrm{a}} \quad$ time

$\Delta p \quad$ externally applied pressure difference

$\Delta p_{1 \text { bar }}$ pressure for normalization

$\Delta p_{\mathrm{v}} \quad$ pressure difference across the throttle valve

$\alpha \quad$ cone angle of closing body and valve seat

$\beta \quad$ lead angle of the screw drive

$\eta \quad$ efficiency of the screw drive

$\mu_{0} \quad$ static frictional coefficient

$\mu \quad$ (kinetic) frictional coefficient 


\section{REFERENCES}

[1] Samson, „Ventile Produktselektor,“14 052019. [Online]. Available: https://www.samson.de/de/produkteanwendungen/produkte/ventile/?tx_solr $\% 5$ Bfilt er $\% 5 \mathrm{D} \% 5 \mathrm{~B} 0 \% 5 \mathrm{D}=$ usage $\% 3 \mathrm{~A} 1$.

[2] Dietrich I, Müller T, Meck M, Zinßer L, Nakhjiri M, Ludwig G, Pelz P (2019) One valve for many purposes - reduced complexity by seperation of functions. 4th International Rotating Equipment Conference - Pumps and Compressors. Sep 2425, 2019, Düsseldorf, Germany.

[3] Moogk D R (2012) Minimum Viable Product and the Importance of Experimentation in Technology Startups. Technology Innovation Management Review, vol. 2, no. 3, pp. 23-26.
[4] DIN EN 60534-3-1:2000-12 Stellventile für die Prozessregelung - Teil 3-1: Abmessungen Einbaulängen von geflanschten Durchgangsventilen und geflanschten Eckventilen (IEC 60534-3-1:2000).

[5] SAMSON Aktiengesellschaft (2019) Typenblatt T 5824. Frankfurt, March 2019.

[6] SAMSON Aktiengesellschaft (2019) Antriebe für elektrische Stellventile. Frankfurt, 2019.

[7] Gomeringer R, Kilgus R, Menges V, Oesterle S, Rapp T, Scholer C, Stenzel A, Stephan A, Wieneke F (2019) Tabellenbuch Metall, 48. Auflage, Europa, 2019.

[8] Grote K.-H., Feldhusen J Dubbel (2005) Taschenbuch für den Maschinenbau mit Tabellen, 22., neubearb. und erw. Aufl. ed. Berlin u.a., Springer, 2005. 